\title{
Status of the first phase of the Alborz Observatory Array: Alborz-I
}

\section{S. Abdollahi; ${ }^{a b}$ M. Bahmanabadi, ${ }^{a b}$ Y. Pezeshkian, ${ }^{a b}$ M. Abbasian Motlagh ${ }^{b}$ and S. Mortazavi Moghaddam ${ }^{b}$}

${ }^{a}$ Department of Physics, Sharif University of Technology

PO Box 11155-9161, Tehran, Iran

${ }^{b}$ Alborz Observatory, Sharif University of Technology

PO Box 11155-9161, Tehran, Iran

E-mail: abdollahi.soheila@gmail.com, bahmanabadi@sharif .edu, pezeshkian@sut.ac.ir, abbasianmotlagh@gmail.com,

mortazavimoghaddam@gmail.com

\begin{abstract}
Alborz-I as the first phase of the Alborz Observatory Array supposed to study the cosmic ray spectrum around the knee at Sharif University of Technology campus, Tehran (1200 m a.s.l). In this paper theoretical results obtained from study of the design features, performance, array response and angular resolution of the Alborz-I consists of 20 plastic scintillation detectors each one with surface area of $0.25 \mathrm{~m}^{2}$ spread over an area of $40 \times 40 \mathrm{~m}^{2}$ are described. Using Monte Carlo simulation of showers, the rate of detected events per day and the trigger probability function, i.e., the probability for an extensive air shower to trigger a ground based array as a function of the shower core distance to the center of array are presented for energies around the knee and zenith angles up to $60^{\circ}$. Moreover, the angular resolution of the array is estimated in the energies of $\sim 3 \times 10^{14} \mathrm{eV}$, which contains the most rate of detected events by the array.
\end{abstract}

The 34th International Cosmic Ray Conference,

30 July- 6 August, 2015

The Hague, The Netherlands

\footnotetext{
* Speaker.
} 


\section{Introduction}

Air shower measurements can determine the arrival direction, energy, and mass of the primary cosmic rays. Study of the primaries provides important information on their origins and acceleration mechanisms and can also explain two observed bends in the cosmic ray spectrum.

Lowest and highest energies at which cosmic rays can be detected by arrays depend on the distance between neighboring detectors and overall size of detector array respectively. Therefore geometry and design of an array have an important role to extend the detectable energy range. Geometrical effects of array layout on the number of detected events, trigger probability and angular resolution are presented here by increasing distance between neighboring detectors and also changing configuration of the array.

Dependence of the trigger probability of array on the primary particle energy $(E)$ and shower core distance from the array center $(r)$ is studied by Monte Carlo simulations of showers convoluted with the array layout and detector response. The trigger probability, known as $P(r, E)$, is also presented as a fundamental parameter to compare different trigger conditions in the both proposed rectangular and cluster layouts. On the other hand, the angular resolution of the array for different trigger conditions and layouts is calculated using a chi-squared minimization algorithm by assuming that the shower front can be approximated by a plane. Two different shower sets simulated by CORSIKA [1] have been applied for these studies.

In this paper, the results from study of the array properties in particular the array layout, trigger conditions, detector response and angular resolution are mentioned in the following sections.

\section{The Alborz-I surface array}

The Alborz-I as the first phase of the Alborz Observatory Array (AOA) is realized to measure the flux, arrival direction and energy of the primary cosmic ray around the knee at Sharif University of Technology (SUT), Tehran $\left(35.72^{\circ} \mathrm{N} 51.33^{\circ} \mathrm{E}\right)$ at $1200 \mathrm{~m}$ a.s.l. It consists of 20 scintillation detectors over an area of about $40 \times 40 \mathrm{~m}^{2}$, and in the next phase, $10 \mathrm{WCDs}$ will be added to it. The used detectors are optimized by a series of experiments and simulations [2, 3].

Each scintillation detector is embedded in a station with a $5 \mathrm{~mm}$ cement ceiling and has a pyramidal galvanized iron light enclosure with $1 \mathrm{~mm}$ wall thickness, housing a $50 \times 50 \times 2 \mathrm{~cm}^{3}$ NE102A scintillator. Scintillation light emitted due to passing a particle through the scintillator (optical photon) is read out from tip of the light enclosure by a $5 \mathrm{~cm}$ PhotoMultiplier Tube (PMT, 9813B) for timing measurements.

Two different array configurations, one cluster and other a $5 \times 4$ rectangular grid, are proposed. As shown in Fig. 1, in the cluster layout, 5 out of 20 detectors arranged on an internal pentagon with side length $5 \mathrm{~m}$ and the rest set on 5 surrounding triangles with the same side length $5 \mathrm{~m}$ which are placed on the corners of an external pentagon with side length about $18 \mathrm{~m}$.

\section{EAS simulation}

To more realistic estimate of the array properties, two different EAS sets which differ in the distribution of energy and zenith angles, simulated by CORSIKA (version 6.9) using QGSJETII [4] and GHEISHA ( $E \leq 80 \mathrm{GeV}$ ) [5] models, have been used. The energy deposition of reached 


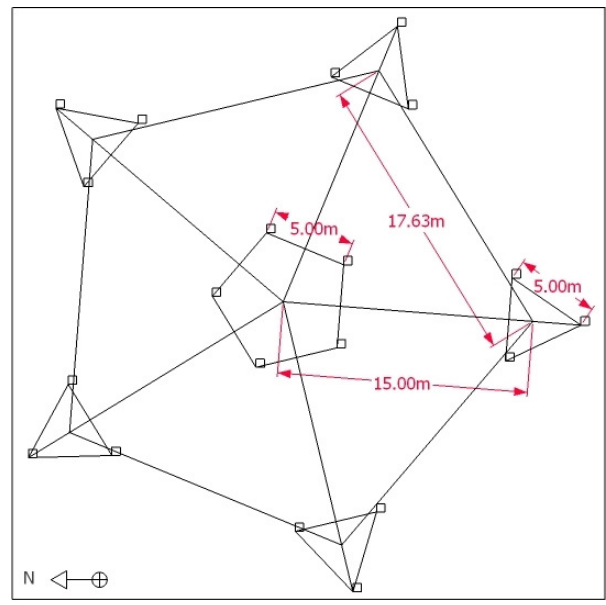

Figure 1: Cluster layout of the Alborz-I array where 20 SDs will be deployed in a square area.

particles to the detectors, passing through the cement stations and the scintillation detectors, and also the optical photons production are calculated using simulated air showers which are used as input for detailed GEANT4 [6] simulations. Finally, the detector response is estimated according to presumptions implemented in the CORSIKA inputs, interaction of particles with the detector material, optical photon processes and the PMT response.

A shower set consists of 3600 extensive air showers with a composition of $88 \%$ proton and $12 \%$ alpha as primary particles have been simulated to reliable estimate of the trigger probability function. In this set, air showers were simulated with zenith angles between $0^{\circ}$ and $60^{\circ}$ distributed as $I \propto \sin \theta \cos \theta \mathrm{d} \theta$, and the energy of primaries discretely distributed in a range between $10^{12} \mathrm{eV}$ and $10^{16} \mathrm{eV}$ in steps of 0.5 in $\log E$. It's worth noting that the cut-off energy of particles kinetic energy is chosen $0.3 \mathrm{GeV}$ for hadrons and muons, and $0.003 \mathrm{GeV}$ for electrons and photons in the CORSIKA and particles blow their energy threshold aren't further tracked. For the both layouts, the core position of any shower is uniformly distributed at ground. A wide square grid including maximum number of 2809 square pixels (a $53 \times 53$ grid), each one with surface area of $49 \mathrm{~m}^{2}$ is considered to study events falling inside and outside the border of the array, while triggered the array. Number of square pixels directly depends on the primary energy of the incident showers (or shower particle disc size). But due to considerable uncertainties for estimation of the core position and energy of the events falling outside the border of the array, events falling on the inner $5 \times 5$ grid (which covers inside the array) are more important for future studies.

In order to increase the statistics of simulated EASs and also to cover different zones of ground with a constant probability in a uniform distribution, the core position of any shower is set in the center of each pixel. Finally, the trigger probability of the array is estimated as a function of core distance and energy for events falling at ground while fulfill the trigger conditions. As mentioned above, a more detailed analysis of the detector response is carried out using GEANT4. Fig. 2 shows the number of optical photons reached to PMT over a range of energies for all particles which considered in the simulations. The range of energies are chosen according to energy distribution of secondary particles obtained by CORSIKA.

Another set of showers includes 12000 proton and alpha showers, with the same previous 

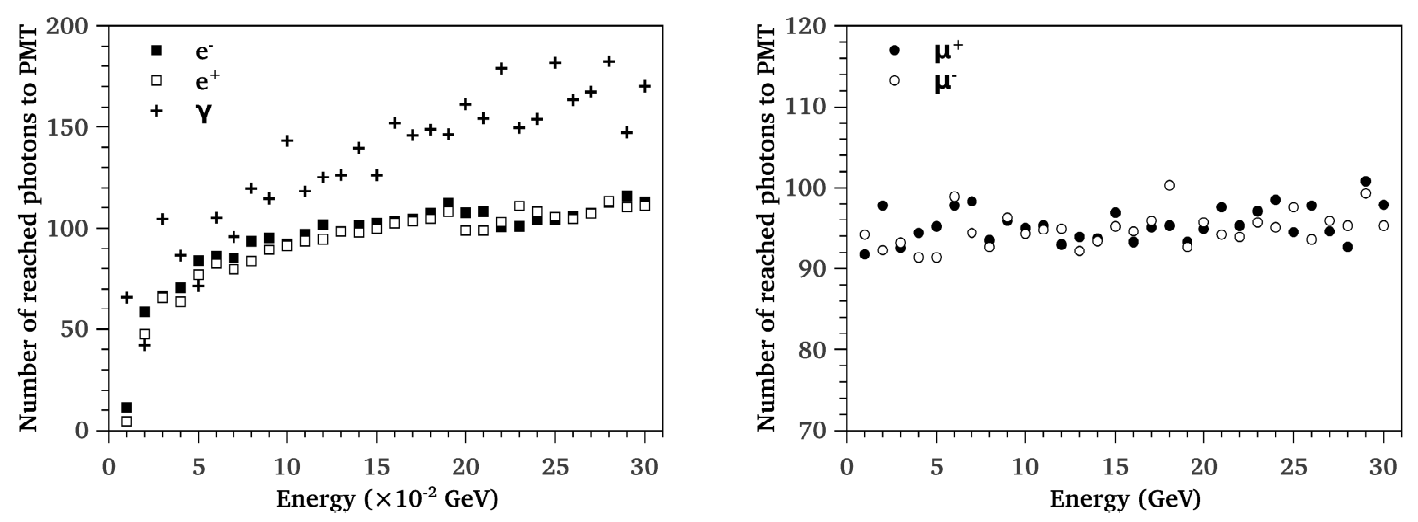

Figure 2: Number of reached photons to PMT for different secondary particles. Energy ranges of more than $97 \%$ gammas, electrons and positrons are blow $1 \mathrm{GeV}$, while for muons are commonly up to $50 \mathrm{GeV}$. As can be seen, number of reached photons to PMT tends to the fix value for each particle at higher energies.

composition, distributed over a continuous energy range from $2 \times 10^{14} \mathrm{eV}$ to $4 \times 10^{14} \mathrm{eV}$ have been generated to estimate the angular resolution of the array. This energy range is selected according to the results of the first simulated showers set which reveal a significant number of recorded events in this range in comparison with other bins of energy. In this set, the zenith angle of showers is selected from $0^{\circ}$ to $60^{\circ}$ in steps of $5^{\circ}$.

\section{Trigger probability function}

The trigger probability of a ground based array depends on many factors such as: i) energy and mass of the primary cosmic ray which initiates an air shower, ii) the type of the array detectors, iii) the trigger condition used to detect air showers, iv) the array layout, v) the geometry of the incoming shower, e.g., its incidence zenith angle and distance of shower core to the array center [7]. These dependencies are shown by the trigger probability function, $P(r, E)$, as a fraction of showers in the energies of interest $(E)$ which fulfill the trigger condition in different bins of shower core distance from the array center $(r)$, as

$$
P(r, E)=\frac{N_{\text {trigger }}(r, E)}{N_{\text {incident }}(r, E)}
$$

In order to optimize the array size in a rectangular configuration, four different array sizes with detector spacings of $1.5 \mathrm{~m}, 3.5 \mathrm{~m}, 7 \mathrm{~m}$ and $14 \mathrm{~m}$ are studied. However the surface area of the site at the SUT campus lets the maximum detector spacing of $7 \mathrm{~m}$ but note that in the last phase, site of the array will be extended. The trigger conditions are provided by randomly at least 4-fold (4 out of 20), 6-fold (6 out of 20), 10-fold (10 out of 20) and finally fully triggering detectors. Because of the small surface of detectors, two last trigger conditions can provide more satisfactory statistical sample of charged particles in the shower disc for future studies on the energy resolution and mass composition. However, for the fully triggering condition the statistic of recorded events decreases significantly. So, as discussed in the next section for the at least 10 -fold condition, the angular resolution accuracy improves significantly rather than at least 4-fold and 6-fold conditions. Thus, in the rectangular layout, the analyses are presented on the at least 10 -fold condition. 

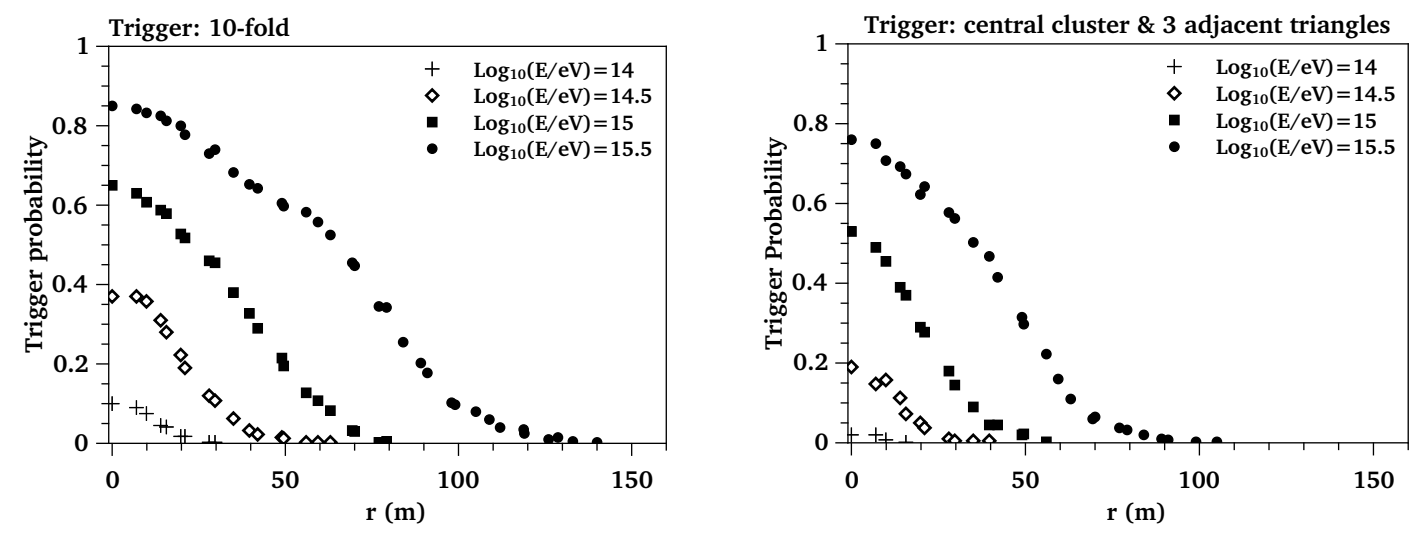

Figure 3: Array trigger probability as a function of shower core distance to the array center $(r)$ for the 10 -fold condition and detector spacing of $7 \mathrm{~m}$ in the rectangular layout (left), and for triggering 5 out of 5 in the central cluster and 3 adjacent triangles in the cluster layout (right) at energies ranging between $10^{14}$ and $10^{16} \mathrm{eV}$ in steps of 0.5 in the logarithmic scale.

Beside the rectangular grid, a cluster layout is also studied. Through several implemented trigger conditions, those conditions with full triggered central cluster and two or three or four surrounding triangles, (i.e., 5 out of 5 in the central cluster and 2 adjacent triangles, 5 out of 5 in the central cluster and 3 adjacent triangles, and finally 5 out of 5 in the central cluster and 4 adjacent triangles) show higher efficiencies, as their trigger probabilities are approximately the same. Therefore, these three trigger conditions are selected: randomly 10-fold, 5 out of 5 in the central cluster, and finally 5 out of 5 in the central cluster and 3 adjacent triangles.

The simulation results has shown that the trigger probability is an increasing function of energy and a decreasing function of distance from the shower core [8]. In Fig. 3 the trigger probability for one of implemented trigger conditions in each layout is shown. As can be seen, the trigger efficiency of the array will be saturated $(\simeq 100 \%)$ for energies above $10^{16} \mathrm{eV}$ and for the shower core distances close to the array center.

A statistical error of the trigger efficiency is defined as the standard deviation of the trigger efficiency at pixels of the square grid which are located at the same distance from the array center. Due to very small achieved uncertainties, error bars are less than the symbol size and aren't shown.

In addition to the trigger probability function, number of triggered events per day in different bins of energy $(N(E, E+\triangle E))$ for the implemented trigger conditions and different array layouts can be calculated by a convolution of the trigger probability function $P(r, E)$ and differential flux of primary cosmic rays $\Phi(E)$ (per area, time, solid angle and energy) (Eq. (4.2)),

$$
N(E, E+\triangle E)=\int_{S} \int_{E}^{E+\triangle E} P(r, E) \Phi(E) d S d E \int d \Omega \int d t
$$

where the intervals are selected according to the default initial conditions in the simulations. $S$ is the surface of a square grid which considered to study showers arriving inside and outside of the array while triggered it. The primary spectrum, $\Phi(E)$, in the knee region is determined with the Tibet air shower array which is located at an almost ideal atmospheric depth for this energy 


\begin{tabular}{|c|c|c|c|c|c|c|c|}
\hline \multirow{2}{*}{$l(\mathrm{~m})$} & \multicolumn{7}{|c|}{$\log (E / e V)$} \\
\hline & 12.5 & 13 & 13.5 & 14 & 14.5 & 15 & 15.5 \\
\hline 1.5 & $450_{-226}^{+320}$ & $349_{-228}^{+297}$ & $3541_{-386}^{+472}$ & $7437_{-916}^{+1039}$ & $6327_{-271}^{+280}$ & $4700_{-254}^{+241}$ & $1397_{-30}^{+28}$ \\
\hline 3.5 & - - - & $116_{-102}^{+133}$ & $2669_{-261}^{+316}$ & $7561_{-906}^{+1029}$ & $6241_{-263}^{+271}$ & $4663_{-253}^{+240}$ & $1391_{-30}^{+28}$ \\
\hline 7 & - - - & - . - & $1283_{-121}^{+143}$ & $5757_{-636}^{+718}$ & $6071_{-249}^{+257}$ & $4772_{-252}^{+239}$ & $1407_{-30}^{+28}$ \\
\hline 14 & - - - & $-\ldots$ & $201_{-28}^{+33}$ & $2453_{-242}^{+267}$ & $5006_{-176}^{+180}$ & $4488_{-231}^{+219}$ & $1413_{-30}^{+28}$ \\
\hline
\end{tabular}

Table 1: Number of events in different bins of energy for 10-fold triggering and different detector spacing $(l)$ in the rectangular grid.

\begin{tabular}{lrrrrr}
\hline \multirow{2}{*}{ Trigger condition } & \multicolumn{5}{c}{$\log (\mathrm{E} / \mathrm{eV})$} \\
\cline { 2 - 6 } 10 out of 20 & 13.5 & 14 & 14.5 & 15 & 15.5 \\
\hline & $1411_{-131}^{+157}$ & $5402_{-573}^{+646}$ & $6072_{-245}^{+252}$ & $4679_{-250}^{+237}$ & $1406_{-30}^{+28}$ \\
$\begin{array}{l}\text { central cluster } \\
\text { central cluster \& 3 adjacent }\end{array}$ & $386_{-59}^{+70}$ & $1324_{-229}^{+258}$ & $1743_{-104}^{+107}$ & $1618_{-125}^{+119}$ & $572_{-17}^{+16}$ \\
triangles & $111_{-24}^{+29}$ & $740_{-132}^{+148}$ & $1488_{-89}^{+91}$ & $1484_{-119}^{+113}$ & $545_{-17}^{+16}$ \\
\hline
\end{tabular}

Table 2: Number of events for different trigger conditions in the cluster layout.

range [8]. In Table 1 the energy distribution of triggered events per day is listed for different array sizes in the rectangular layout and for 10 -fold condition.

A similar analysis for the different trigger conditions in the cluster layout is also performed. As can be seen in Table 2, the maximum rate of triggered events happens in $3 \times 10^{14} \mathrm{eV}$, similar to the rectangular grid with detector spacing 7 and $14 \mathrm{~m}$. It should be noted that the errors are due to systematic uncertainties of the cosmic ray spectrum which is used in the Monte Carlo simulations to estimate the rate of triggered events per day.

\section{Angular resolution}

The angular resolution of the Alborz-I is discussed by considering the second shower set including 12000 showers distributed over a continuous range from $2 \times 10^{14} \mathrm{eV}$ to $4 \times 10^{14} \mathrm{eV}$ with $\theta$ between $0^{\circ}$ and $60^{\circ}$ in steps of $5^{\circ}$. This energy range is selected according to receiving maximum number of events in $E=3 \times 10^{14} \mathrm{eV}$.

The arrival direction of a shower is determined by an optimized fit of a plane to the arrival times of particles reached to the detectors. Using a chi-squared minimization algorithm the angular resolution of the array for different trigger conditions and layouts is calculated. The angular resolution is directly related to the accuracy of timing measurement. Therefore to provide more realistic 

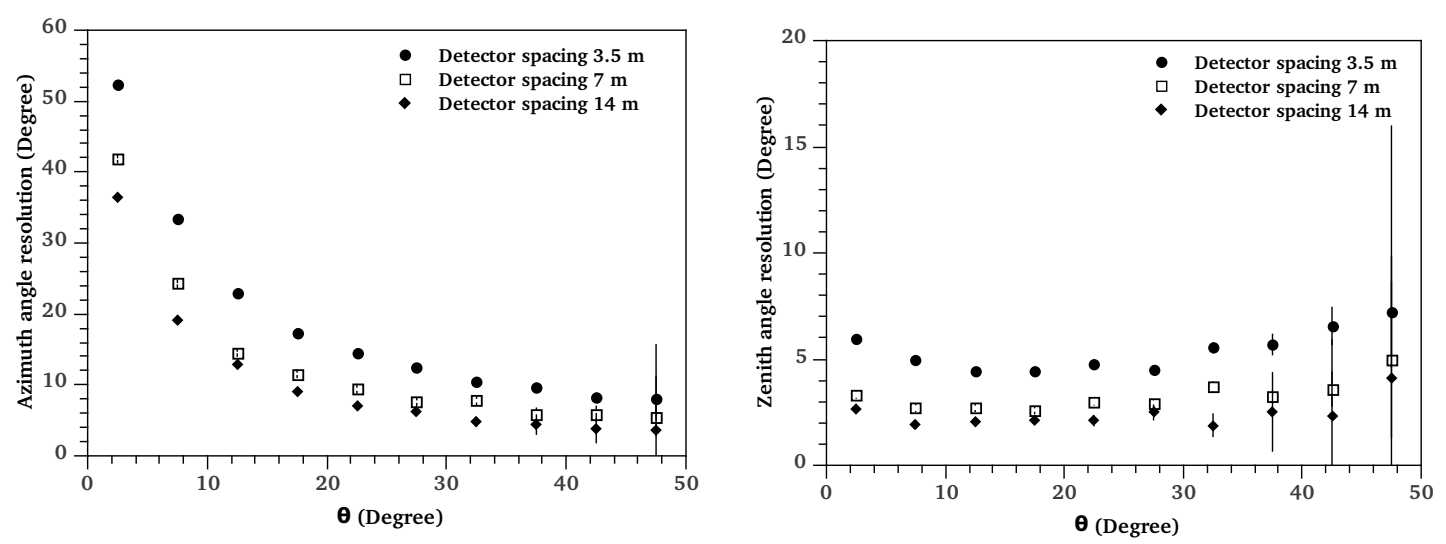

Figure 4: Azimuth angle resolution (left) and zenith angle resolution (right) as a function of primary's zenith angle in the rectangular layout for the 10 -fold condition.

results, the uncertainty on the timing measurement is implemented in the simulation and core position of showers are randomly distributed at all over the array surface. The uncertainty on the timing measurement by the Alborz-I instruments is obtained by series of coincidence experiments as

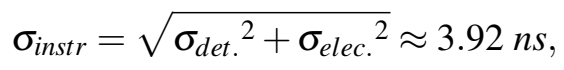

where $\sigma_{\text {det. }}$ and $\sigma_{\text {elec. }}$ are the detector and the electronic uncertainties, respectively.

Fig. 4 shows that the angular resolution is an increasing function of the array size and although the angular resolution improves for the detector spacing $14 \mathrm{~m}$, but there is a small difference between $7 \mathrm{~m}$ and $14 \mathrm{~m}$ detector spacing. In addition, as mentioned above, the site of array limits detector spacing to $7 \mathrm{~m}$. As illustrated in Fig. 5, the angular resolution improves significantly in the cluster layout in comparison with the rectangular grid with triggering 10-fold and detector spacing $7 \mathrm{~m}$. Through the three selected trigger conditions in the cluster layout, those which include fully triggered central cluster show almost the same angular resolutions. The errors represent statistical uncertainties, however, as mentioned above the systematic uncertainties which are due to the time resolution of the array instruments is included in the analysis.

\section{Conclusion}

Monte Carlo studies based on the CORSIKA program have been performed on the Alborz-I array characteristics. The trigger probability function, angular resolution and energy distribution of recorded events per day have been estimated for two layouts and different trigger conditions.

The results show the primary energy and core location dependences of the trigger probability. As can be seen, the saturation $(P(r, E) \simeq 100 \%)$ will be happened for $E>10^{16} \mathrm{eV}$.

In the rectangular layout, four different trigger conditions and four array sizes were considered. Study on the angular resolution showed that it obviously improves as the array size and number of triggered detectors increase. But limitation in the surface area of the array and on the other hand, the significant decrease in recording events for fully triggering of the array (20-fold triggering 

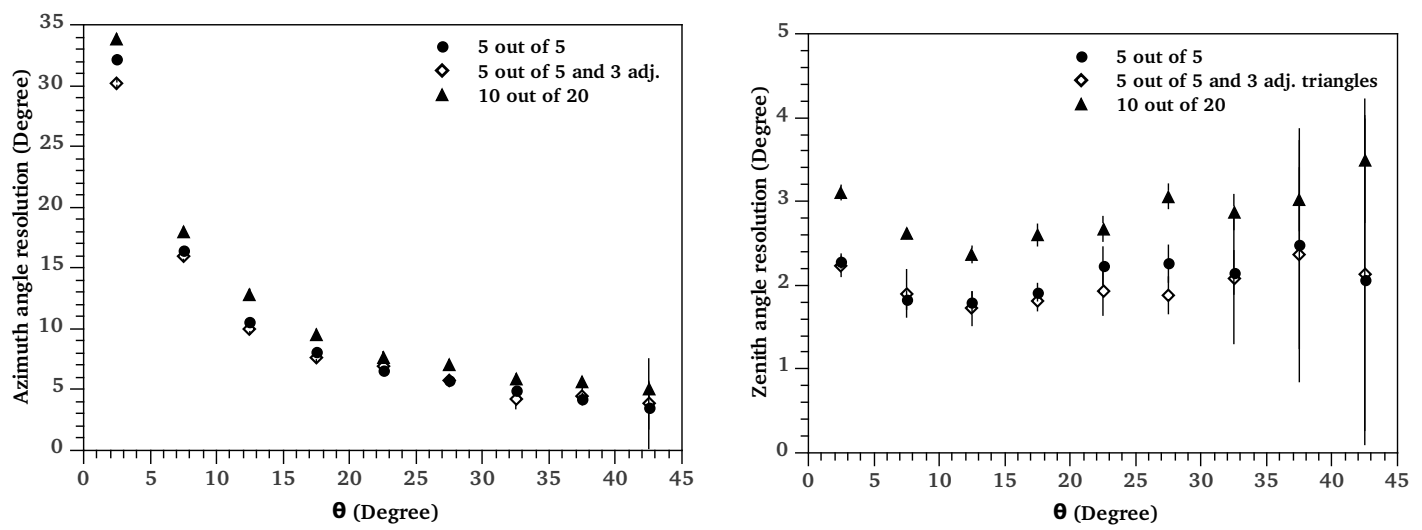

Figure 5: Azimuth angle resolution (left) and zenith angle resolution (right) as a function of primary's zenith angle for the cluster layout.

condition) made us consider the results for detector spacing $7 \mathrm{~m}$ and the triggering of at least 10 -fold as default conditions to compare with the results of the pentagon layout.

In the pentagon layout, the implemented trigger conditions which include fully triggered central cluster together with some of the adjacent triangles yield approximately the same efficiency for each primary energy. The angular resolution of the pentagon layout also improves significantly in comparison with the rectangular grid with $7 \mathrm{~m}$ detector spacing and 10-fold trigger condition.

\section{References}

[1] D. Heck et al., CORSIKA: a Monte Carlo code to simulate extensive air showers, Report FZKA 6019 (1998), Forschungszentrum Karlsruhe

[2] S. Mortazavi Moghaddam et al., Optimization of dimensions and inner surface of water Cherenkov detector with one photomultiplier tube (PMT) for the Alborz observatory air shower array, Astroparticle physics 35 (2012) 792-796

[3] Y. Pezeshkian et al., Scintillation detectors of Alborz-I experiment, Nucl. Instr. Meth. A 773 (2015) $117-123$

[4] N.N. Kalmykov et al., Quark-gluon-string model and EAS simulation problems at ultra-high energies, Nucl. Phys. B (Proc. Suppl.) 52 (1997) 17-28

[5] H. Fesefeldt, The simulation of hadron showers, RWTH Aachen Report, Report No. PITHA 85/02 (1985)

[6] R. Brun and F. Carminati, GEANT detector description and simulation tool, CERN program library long writeup W5013, (1993)

[7] The Pierre Auger Collaboration, The lateral trigger probability function for the ultra-high energy cosmic ray showers detected by the Pierre Auger Observatory, Astroparticle physics 35 (2011) 266-276

[8] M. Amenomori et al., The cosmic ray energy spectrum between $10^{14.5}$ and $10^{16.3} \mathrm{eV}$ covering the Knee Region, ApJ 461 (1996) 408-414 